\title{
„Rationale“ vs. (?) „kommunikative“ Planungsmethoden: Theoretische Ausgangspunkte, empirische Befunde aus Experimenten und Überlegungen zur Weiterentwicklung am Beispiel der Nutzwertanalyse
}

\author{
Christian Diller ${ }^{1} \cdot$ Sarah Oberding ${ }^{2}$ \\ Eingegangen: 20. Oktober 2017 / Angenommen: 1. November 2018 / Online publiziert: 26. November 2018 \\ (c) Die Herausgeber 2018
}

\section{Zusammenfassung}

Ziel des Beitrags ist es, auf der Basis einer vereinfachten theoretischen Fundierung empirische Hinweise auf den Charakter von unterschiedlichen Planungsmethoden zu gewinnen, um daraus Folgerungen für ihre Weiterentwicklung zu ziehen. Dabei wird der Frage nachgegangen, inwieweit sich unterschiedliche Verständnisse von Planungsrationalität auch in unterschiedlichen Planungsmethoden ausdrücken. Dazu wird zunächst die Debatte um den Rationalitätsbegriff in der Planung wiedergegeben, die sich vor allem auf die Dichotomie zwischen analytischer Zweck-Mittel-Rationalität einerseits und kommunikativer Rationalität andererseits zuspitzen lässt. Hierauf aufbauend werden empirische Ergebnisse aus einem Forschungsvorhaben vorgestellt, in dem verschiedene Planungsmethoden auf die drei Hauptdimensionen „,rational“, „kommunikativ“ und „kreativ“ hin bewertet wurden. Daran anknüpfend wird am Beispiel der klassischen Planungsmethode der Nutzwertanalyse dargelegt, ob und wie vermeintlich rationale Planungsmethoden bislang im Hinblick auf den Communicative Turn in der Planung weiterentwickelt wurden. In der noch systematischeren kommunikativen Ausgestaltung bewährter rationaler Planungsmethoden wird im Fazit auch ein wichtiges und praxisrelevantes Feld der Planungsforschung erkannt.

Schlüsselwörter Rationalität · Kommunikation $\cdot$ Planungsmethoden $\cdot$ Experimente $\cdot$ Nutzwertanalyse

\section{Rational vs. (?) communicative planning methods: Theoretical starting points, empirical findings from experiments and considerations for development of methods on the example of the utility-analysis}

\begin{abstract}
The aim of the article is, based on focused theoretical considerations, to find empirical results concerning the character of several planning methods to find conclusions for their further development. Examined is the question if several understandings of planning rationality are expressed in several planning methods. Firstly, the debate on the term of rationality in planning is reflected, which can be pointed to the dichotomy between means-and-ends-rationality on one hand and communicative rationality on the other. Based on this, empirical findings of a research project are presented, where several planning methods were valued on three main dimensions: "rational", "communicative" and "creative". Connected to this, on the example of the useful value analysis is examined if rational planning methods were developed towards the "communicative turn" in planning. In the conclusion a more systematic communicative design of proven rational planning methods is seen as an important and practical relevant field of planning research.
\end{abstract}

Keywords Rationality $\cdot$ Communication $\cdot$ Planning methods $\cdot$ Experiments $\cdot$ Useful value analysis

Prof. Dr. Christian Diller

christian.diller@geogr.uni-giessen.de

Sarah Oberding

sarah.oberding@lahn-dill-kreis.de
Professur für Raumplanung und Stadtgeographie, Justus-Liebig-Universität Gießen, Senckenbergstraße 1, 35390 Gießen, Deutschland

2 Schulabteilung, Bildungsmonitoring, Lahn-Dill-Kreis, Karl-Kellner-Ring 51, 35576 Wetzlar, Deutschland 


\section{Theoretische Bezugspunkte: Die Veränderungen des Rationalitätsbegriffs in der Planung}

Der Begriff der Rationalität ist für jegliche Form von Planung, also auch die Raumplanung, zentral und bestimmt das Planungsmodell maßgeblich. Jede Planung braucht daher ein Grundverständnis von Rationalität oder basiert doch zumindest implizit auf einem solchen. Planung ist der Versuch, die Rationalität zukünftigen Handelns zu steigern (Bäcker 1996: VII, 15 ff.). In der Planungstheorie, aber auch in anderen Disziplinen wie der Philosophie oder den Wirtschaftswissenschaften, wurde die Debatte um den Rationalitätsbegriff von den 1960er-Jahren bis in die frühen 1980er-Jahre vermutlich am intensivsten geführt. In der Philosophie verdrängte der Rationalitätsbegriff teilweise sogar den Begriff der Vernunft (Lenk/Spinner 1989: 1).

Ausgangs- und Reibungspunkt der Debatten um Rationalität ist nach wie vor das rationale Planungsmodell, das zunächst die Zweck-Mittel-Relation in den Vordergrund stellt. Es geht von einem eigennützig denkenden und zweckrational handelnden Homo Oeconomicus aus. Seine Rationalität basiert auf wahrscheinlichkeitstheoretischen Überlegungen und den ethischen Prinzipien des Utilitarismus. Eine Entscheidung ist dann rational, wenn innerhalb des Prozesses zur Entscheidungsfindung die Handlungsalternative ausgewählt wird, die im Hinblick auf die vorgegebene Zielfunktion optimal ist, wobei auch der Umgang mit den Aspekten Sicherheit, Risiko und Unsicherheit optimiert wird (Bäcker 1996: 129). Der Systemansatz der Planungstheorie der „ersten Generation“ (Rittel 1992: 39) ist diesem Rationalitätsprinzip verpflichtet. Der Planungsprozess besteht in einer klaren sequenziellen Abfolge aus Problemdefinition, Informationssammlung und -analyse, Entwicklung der Lösungen, vergleichender Bewertung der Lösungen und Auswahl sowie Implementierung und Evaluation der ausgewählten Lösung und gegebenenfalls ihrer Modifikation.

Die Kritik an diesem Rationalitätsbegriff bezieht sich zunächst einmal nicht auf seinen normativen Gehalt, also wie Denken, Handeln und Planen ausgerichtet sein soll, sondern vielmehr darauf, wie tatsächlich agiert wird und ob die unterstellten Voraussetzungen überhaupt gegeben sind. Das klassische rationale Planungsmodell geht von zumindest drei Voraussetzungen aus: vollständige Information über alle verfügbaren Alternativen, widerspruchsfreie Ziele und ausreichende Mittel, die gesetzten Ziele umzusetzen (Baum 1996: 127; Siebel 2006: 203). Diese Voraussetzungen treffen, wie Rittel (1992) eindrucksvoll ausführt, lediglich für einfach strukturierte ,gutartige“ Probleme (Bäcker 1996: 127) und Aufgabenstellungen aus den klassischen Ingenieurwissenschaften zu, nicht aber für die Raumplanungsprozesse, die auch immer politisch sind. Hier geht es in der Regel um „bösartige“ Probleme. Diese sind einmalig und haben keine überschaubare Menge möglicher Lösungen und es ist nicht zu entscheiden, wann sie gelöst sind. Lösungen für „,bösartige“ Probleme können daher nicht „richtig“ oder "falsch“, sondern bestenfalls gut oder schlecht sein und sie sind "One-shot-Operationen“" ohne Wiederholungsmöglichkeit (Rittel 1992: 13).

Diese und andere Kritikpunkte am klassischen Rationalitätsbegriff führten in der Planungstheorie, aber auch in anderen Disziplinen wie der Betriebswirtschaft (Bäcker 1996: 3), zu Verunsicherungen: „After Rationality, what?“” (Alexander 1984). Im Ergebnis weitete sich das Spektrum von Rationalitätsbegriffen deutlich auf. In der Philosophie wurden umfassende Klassifikationen von Rationalität vorgelegt. Elster (1982) gliedert Rationalität beispielsweise anhand von sechs Dichotomien, Lenk und Spinner (1989) unterscheiden gar 22 Rationalitätsbegriffe. Rationalität kann sich demnach nicht nur auf die Effizienz der Zweck-MittelRelation und auf die logische Konsistenz von Argumenten, Entscheidungen und Handlungen und deren intersubjektive Nachvollziehbarkeit, sondern auch normativ auf die gesetzten Ziele selbst beziehen. Rationalität ist immer ein normatives Konzept, um Probleme zu lösen und mit der Fähigkeit zur Selbstreflexion und zu Lernprozessen verbunden (Bäcker 1996: $15 \mathrm{ff}$.).

In der Planungstheorie wurde als alternatives Planungsmodell zu dem klassischen rationalen Modell das Konzept der „Bounded Rationality“ (Simon 1991) entwickelt, das dem Umstand der beschränkten Information Rechnung trägt und zufriedenstellende anstelle optimaler Lösungen als einzuhaltende Norm formuliert, und der Inkrementalismus, der das schrittweise pragmatische und reaktive ,Durchwursteln ${ }^{\text {* }}$ bei der Lösung von Planungsproblemen zum Paradigma erhebt und mit seinem adaptiven Strategiemodell den Kontrapunkt zum linearen Strategiemodell der Rationalisten formuliert (Wiechmann 2008: 60). Die meisten seitdem vorgeschlagenen Modelle des Planungsprozesses (für einen Überblick vgl. Hoffmann/Oberding 2017) stellen Hybridformen des klassischen rationalen und des inkrementellen Planungsmodells dar. Insbesondere der „Perspektivische Inkrementalismus“" (Ganser/Siebel/Sieverts 1993: 113) hat in Deutschland in den 1990er-Jahren viel Aufmerksamkeit erhalten. Auch der in den letzten Jahren diskutierte Begriff der „strategischen Planung“ (Wiechmann/Hutter 2010) versucht, Antworten auf die Differenz zwischen dem Anspruch der Rationalität und den tatsächlichen begrenzt rationalen Planungsentscheidungen zu finden (Wiechmann 2008: 15).

Ein gewisser Minimalkonsens zum Rationalitätsbegriff lässt sich sicherlich im Rückblick auf seinen begriffsgeschichtlichen Ursprung formulieren. Rationalität wird meist als Konzept im Sinne der Nachvollziehbarkeit von Gründen gesehen und ist insofern immer etwas Intersubjektives. Daher wurde in der Planungstheorie das Moment der Kommunikation aller Akteure als zentrales Merkmal der Herstel- 
lung von Rationalität immer stärker betont. Bereits Rittels Ansatz der Planung der ,2. Generation“ betonte die Bedeutung partizipativer und kommunikativer Elemente und reduzierte die Bedeutung des Expertenwissens und der Wissenschaft zugunsten eines Pluralismus der Urteile, der auch auf Intuitionen basiert (Rittel 1992: $49 \mathrm{ff}$.). Dieses Moment der kommunikativen Verständigung wurde in Schönwandts Planungstheorie der „3. Generation“ (Schönwandt 2002) noch weiter akzentuiert. International wird seit den 1990erJahren sogar von einem „Communicative Turn“ (Healey 1992) gesprochen: Dieser versteht Planung vor allem als soziale Interaktion und formuliert ein kommunikatives Planungsmodell, das den herrschaftsfreien Diskurs im Sinne von Habermas und somit die „kommunikative Rationalität“ zum normativen Paradigma der Planung erhebt.

Wie Siebel (2006: $203 \mathrm{ff}$.) zusammenfasst, haben in der Raumplanung mehrere Formen von Rationalität Berechtigung, darunter sowohl die lineare Zweck-Mittel-Rationalität als auch die kommunikative Rationalität. Diese einzelnen Modelle haben zwar jeweils Schwächen: Das klassische rationale Modell versagt, wenn es um Innovation und Motivation geht, da es in der strikten Anwendung von Prinzipien wie Zweck-Mittel-Rationalität und linearer Lösungsentwicklung die Suche nach Lösungen zu sehr einschränken kann. Das offene, kommunikative Konsensmodell scheitert häufig an strukturellen Machtasymmetrien. Die jeweiligen Schwächen können jedoch kompensiert werden, wenn die unterschiedlichen Rationalitätsbegriffe der Planung flexibel in unterschiedlichen Situationen und zu unterschiedlichen Problemstellungen zur Anwendung kommen (Siebel 2006: $208 \mathrm{f}$.). ${ }^{1}$ Das linear-rationale Strategieverständnis hat also nach wie vor seinen Wert zumindest als normative Messlatte, als Ausgangspunkt, um Abweichungen von ihm zu analysieren; es darf jedoch nicht als Verhaltensdeskription missverstanden werden (Wiechmann 2008: 26, 43, $247 \mathrm{f}$.).

Der klassische Rationalitätsbegriff ist damit jedoch nicht ad acta gelegt, sondern hat im Alltag von Planern als Leitidee ein hohes Beharrungsvermögen bewiesen. Nicht wenige Modelle des Planungsprozesses (z. B. Bryson 2003) bleiben trotz eingebauter Rückkoppelungsschleifen letztlich doch dem linear-rationalen Ansatz verpflichtet (Wiechmann 2008: 154). Für dieses Beharrungsvermögen des klassischen Rationalitätsparadigmas gibt es mehrere Gründe: Es bietet in unübersichtlichen Situationen psychologische Handlungssicherheit an, es bestätigt die planerische Profession und es fordert die wissensbasierte Entscheidung (Dalton 1986; Baum 1996: 134).

\footnotetext{
1 Als weitere Form der Rationalität neben der klassischen und der kommunikativen Rationalität kommt für Siebel (2006: 203 ff.) noch die Form der Rationalität des revidierbaren Irrtums hinzu, wonach Planung rational ist, wenn sie erlaubt, dass Irrtümer revidiert werden können, was vor allem im Hinblick auf ökologische Faktoren bedeutsam ist.
}

Für den Kontext der Analyse von Planungsmethoden bedarf die Debatte um das rationale und das kommunikative Planungsmodell allerdings einer Ergänzung, nämlich die emotional-kreative Dimension. Diese Dimension, die vor allem in Kreativitätsmethoden eine hohe Bedeutung hat, wird von Schubert (2014) in die Planungstheoriedebatte eingebracht und hat durchaus ihre Begründung: Wie aus der Psychologie und der Unternehmensführungspraxis bekannt ist (Goleman 1996; von Kanitz 2015), alleine schon deshalb, weil die Verarbeitungskapazität des Bewusstseins dem Unterbewusstsein unterlegen ist. Von daher gilt es, gute Planung auch als Anwendung der Erfahrung, wann rational und wann emotional-intuitiv zu handeln ist, zu verstehen.

In der nachfolgend in Kapitel 2 dargestellten Untersuchung wird der Frage nachgegangen, ob und wie sich die unterschiedlichen Planungsmodelle in unterschiedlichen Planungsmethoden zeigen - zugespitzt: Gibt es rationale auf der einen und kommunikative Planungsmethoden auf der anderen Seite? Die Überlegungen gehen von dem von Siebel (2006) als Kompromissmodell formulierten Vermittlungsversuch zwischen einem Planungsmodell der analytischen Zweck-Mittel-Rationalität auf der einen und dem Modell der kommunikativen Rationalität auf der anderen Seite aus, das um den Aspekt der emotionalen Rationalität erweitert wird. In Kapitel 2.1 werden dazu zunächst Hypothesen zu Dimensionen von verschiedenen Planungsmethoden formuliert. In Kapitel 2.2 wird ausführlicher auf die Methodik der empirischen Untersuchung eingegangen, wobei auch der Stand der Forschung zu experimentellen Forschungsmethoden kurz gestreift wird. In Kapitel 2.3 werden die Ergebnisse der empirischen Untersuchung vorgestellt. Vertieft wird dann in Kapitel 3 auf eine etablierte rationale Methode, die Nutzwertanalyse, eingegangen. Es wird herausgearbeitet, wie sie ,kommunikativ“ weiterentwickelt werden kann. In Kapitel 4 werden schließlich Endhypothesen der Untersuchung formuliert und es wird ein Ausblick auf die weitere Forschung gegeben.

\section{Zur Messung der Bewertung der rationalen, kommunikativen und kreativen Dimension von Planungsmethoden mithilfe von Experimenten}

Die Frage dieses Kapitels ist, inwieweit sich die unterschiedlichen Verständnisse von Rationalität, insbesondere das klassische Verständnis analytischer Rationalität vs. kommunikativer Rationalität, ergänzt um den Aspekt der emotionalen Kreativität in den in der Raumplanung eingesetzten Planungsmethoden ausdrücken. Zugespitzt: Gibt es „,rationale“ Methoden auf der einen und „kommunikative“ sowie „kreative“ Methoden auf der anderen Seite und hat 
sich deren Bedeutung im Laufe der Zeit verändert? Bereits ein Blick in die Lehrbücher der Raumplanung aus unterschiedlichen Jahrzehnten scheint diese Frage zu bejahen. Das noch in den 1970er-Jahren entwickelte umfassende Lehrbuch von Meise und Volwahsen (1980) enthält fast ausschließlich standardisierte, quantifizierende Methoden, die dem klassischen Begriff analytischer Rationalität zu entsprechen scheinen: etwa Prognosen, Kosten-NutzenAnalysen und Nutzwertanalysen. Das Hauptthema bei der Anwendung war vor allem die Verarbeitung großer Informationsmengen, was zu dieser Zeit durch neue Datenverarbeitungssysteme ermöglicht wurde. Akteure der Planung wurden damals nicht thematisiert. Das derzeit umfassendste und relativ aktuelle Lehrbuch der Raumplanung von Fürst und Scholles (2008a) behandelt zwar auch diese Methoden, es wird aber Methoden, die der kommunikativen Akteureinbindung dienen wie Metaplantechnik und Mind Mapping, ebenfalls breiter Raum gegeben. Andere Lehrbücher behandeln sogar nur ausschließlich diese Methoden der kommunikativen und kreativen Prozessgestaltung (z. B. Bischoff/Selle/Sinning 2005).

Empirische Befunde deuten darauf hin, dass in Deutschland der „Communicative Turn“ (Healey 1992) stattgefunden hat (vgl. Diller 2010). Im Rahmen einer bundesweit repräsentativen Befragung (Zufallsauswahl, $\mathrm{N}=1.628$ ) von Raumplanern in verschiedenen Institutionentypen und Planungsformen wurde ermittelt, dass als vermeintlich „kommunikativ" bezeichnete Methoden (wie z. B. Moderationsmethoden, Planspiele) demnach zum einen insgesamt stärker verbreitet waren als ,analytisch-nationale“ Methoden (wie z.B. die Nutzwertanalyse) und dass diese zum anderen bei den jüngeren befragten Planenden noch stärker verbreitet waren als bei den älteren.

Den Rahmen der nachfolgend beschriebenen Untersuchung, die auch die unterschiedlichen Rationalitätsdimensionen von Planungsinstrumenten zum Gegenstand hatte, bildete das Forschungsprojekt ,Raumplanungsmethoden als kollektive Handlungsressource im Communicative Turn“ (2013-2016) der Deutschen Forschungsgemeinschaft (DFG). Nachfolgend werden Hypothesen, Methodik und Ergebnisse zur Anwendung und Bewertung von Planungsmethoden im Rahmen von Experimenten vorgestellt.

\subsection{Ausgangshypothesen}

Für die Formulierung der Hypothesen wurden die in den in Kapitel 1 diskutierten Planungsmodellen enthaltenen Rationalitätsverständnisse präzisiert. Unter der Annahme, dass Planungsmethoden der Aktivierung mehrdimensionaler Akteurpotenziale dienen können, lassen sich zumindest (zu komplexeren Intelligenzbegriffen vgl. Süß/Beauducel 2011) drei zentrale Bewertungsdimensionen aus der Intelligenzforschung unterscheiden (Sternberg 2002; Jetter/Skrotzki
2005; Rost 2010; Winkler/König/Kleinmann 2010) und für das Thema der Bewertung von Planungsmethoden fruchtbar machen:

- Rationalität: Analytische Intelligenz primär zur Problemstrukturierung, Problemlösung und Entscheidungsfindung; der Begriff kommt dem klassischen Intelligenzbegriff am nächsten;

- Kommunikation: Praktische Intelligenz zur Umsetzung von Lösungen, wobei vor allem kommunikative Intelligenz eine entscheidende Rolle spielt;

- Kreativität: Schöpferische Intelligenz zur Suche nach den ,guten' Problemen und zur Generierung von innovativen Lösungen.

Ausgangspunkt ist zunächst die aus der Planungstheoriedebatte abgeleitete Dichotomie „Rationalität“” vs. „Kommunikation“. Diese Erweiterung um die Dimension der Kreativität ist inspiriert durch die Intelligenzforschung. Auch hier hat sich über die Jahrzehnte das Verständnis des ursprünglich auf die Dimension der analytischen Rationalität beschränkten Intelligenzbegriffs erweitert (Sternberg 2002) und die kommunikative Dimension der Intelligenz einen deutlich höheren Stellenwert erhalten. Daher kommt als dritte Hauptdimension die kreative Intelligenz hinzu, welche in der Raumplanung durchaus eine Entsprechung in den so bezeichneten Kreativitätsmethoden hat, wenngleich die Planungstheoriedebatte dem Kreativitätsbegriff keine so zentrale Rolle einräumt wie dem Rationalitätsbegriff und der Kommunikation (Schubert 2014).

Aus den in Kapitel 1 dargestellten Überlegungen werden folgende Ausgangshypothesen zum Unterscheiden der Planungsmethoden abgeleitet:

H1: Planungsmethoden lassen sich in ihren Bedeutungsanteilen der rationalen, kommunikativen und kreativen Dimension und damit in ihren Möglichkeiten, die entsprechenden Potenziale der Akteure in Planungsprozessen zu aktivieren, eindeutig unterscheiden.

H2: Für ausgewählte Planungsmethoden verhält sich die Unterscheidung wie folgt:

- Netzplantechnik und Nutzwertanalyse sind ,rationale Methoden

- Planspiel und World Café sind ,kommunikative“ Methoden

- Mind Mapping ist eine ,kreative ' Methode

- Die SWOT-Analyse und der Strategic Choice Approach sind Methoden sowohl mit hohen rationalen als auch kommunikativen Anteilen

Die Auswahl der in H2 genannten Methoden erfolgte letztlich pragmatisch. Es handelt sich um Methoden, die innerhalb der Lehre am Institut für Geographie der Jus- 
tus-Liebig-Universität Gießen vermittelt werden. Sie präsentieren ein breites Spektrum der Planungsmethoden mit vermutlich unterschiedlichen Akzenten auf den drei Dimensionen ,rational', ,kommunikativ` und ,kreativ“.

\subsection{Methodik}

Die diesem Beitrag zugrunde liegende empirische Untersuchung wurde an der Justus-Liebig-Universität Gießen durchgeführt. Mit dortigen Studierenden der Geographie wurden fragebogenbasierte Experimente ausgerichtet. Diese Methodik wird nachfolgend erläutert.

\subsubsection{Experimentelle Methoden in der Planungsforschung: Vor- und Nachteile}

Bei den für die raumbezogenen Wissenschaften relevanten Experimenten in der Forschung lassen sich Laborexperimente von Feldexperimenten unterscheiden. Feldexperimente finden im Unterschied zu Laborexperimenten unter den Bedingungen der natürlichen unkontrollierten Umwelt statt, in die sich der Forscher als mehr oder minder aktiver Beobachter einzufügen hat. Sie sind in raumbezogenen Wissenschaften gängig. Laborexperimente dagegen sind in den Planungswissenschaften eine bislang relativ selten eingesetzte Forschungsmethode. Dennoch gibt es einige Beispiele dafür, auch solche, bei denen es ebenfalls um Planungsmethoden bzw. Instrumente geht. So führte etwa Te Brömmelstroet (2015) vor dem Hintergrund der methodischen Fragestellung nach den Potenzialen von Experimenten einen Versuch durch, in dem die Effekte von Planungsunterstützungssystemen (Planning Support Systems, PPS) getestet wurden. Hemberger (2014) entwickelte ein Trainingsprogramm zur Schulung für die Lösung komplexer Planungsaufgaben. Zur Evaluation des Trainingsprogrammes wurde ein laborartiges Design eingesetzt, bei dem studentischen Gruppen mit und ohne Schulung in dem Trainingsprogramm Planungsprobleme zur Lösung (Erarbeitung eines Strategiepapers) vorgelegt wurden. Gill, Lange, Morgan et al. (2013) untersuchten im Rahmen der Erarbeitung eines Konzepts zum Umgang mit Hochwasser den Einsatz unterschiedlicher Medien im Planungsprozess. Stamps, Nasar und Hanyu (2005) ermittelten die möglichen Wirkungen städtebaulicher Gestaltungsrichtlinien, Shipley und Michela (2006) untersuchten in laborähnlichen Experimenten die Wirkungen der unterschiedlichen Ausgestaltung stadtplanerischer Visionen und Leitbilder. Julian, Reischl, Carrick et al. (1997) führten Partizipationsverfahren in drei Gruppentypen mit je drei Themen durch. Die Gruppentypen unterschieden sich vor allem in der Intensität der Partizipation, die von längeren Workshops, bei denen die Probanden auch in Kontakt zu den Planern und Politikern kamen (in einer eher laborartig kontrollierten Situation), bis hin zu einfacher Beteiligung per E-Mail (in einer eher unkontrollierten Situation) reichte.

Laborexperimente haben gegenüber Feldstudien den Vorteil, unter kontrollierbaren Bedingungen Unterschiedsund Wirkungshypothesen relativ stringent mit einem günstigen Aufwand-Nutzen-Verhältnis prüfen zu können. Sowohl die interne Validität (Stringenz des kausalen Rückschlusses) als auch ihre Realibilität (Kontrollmöglichkeit von Messfehlern) sind daher höher als in Feldstudien. Die Hauptkritik an Experimenten gegenüber den üblichen feldorientierten Erhebungsverfahren der empirischen Sozialforschung ist die einer geringeren externen Validität: Die Laborsituation ist gegenüber der realen Welt künstlich und entsprechend lassen sich auch Erkenntnisse nicht 1:1 übertragen. Die Vorinformiertheit und das Verhalten von Probanden sind in experimentellen Situationen oft anders als in der realen Welt. Auch im Hinblick auf die Reliabilität bzw. Reproduzierbarkeit sind experimentelle Designs nicht unproblematisch, alleine schon deshalb, weil Lerneffekte der Forscher, eventuell auch der Probanden aus einzelnen Versuchen resultieren (Te Brömmelstroet 2015: 183, 195). Allerdings lassen sich durch das Versuchsdesign und das Durchführen von Experimenten mit anderen Anordnungen sowie die Verknüpfungen mit Beobachtungen in der realen Welt Rückschlüsse auf die Übertragbarkeit der Laborergebnisse erzielen (Kanitsar/Kittel 2015: $382 \mathrm{f}$.).

\subsubsection{Ziel und Methodik der Untersuchung}

Die nachfolgend dargestellten Laborexperimente haben für die Fragestellung nach den Dimensionen von Planungsmethoden vor allem den Vorteil der relativ hohen internen Validität zur Überprüfung von Hypothesen. Ziel ist es, Planungsmethoden im Hinblick auf ihre rationale, kommunikative und kreative Dimension mithilfe eines standardisierten Fragebogens zu bewerten. Die Erfahrungen, die diese Bewertung ermöglichen, sammelten Studierende kurz vorher im Rahmen der Lösung von Planungsaufgaben, in denen jeweils eine von sieben Planungsmethoden eingesetzt wurde. Abbildung 1 zeigt das Vorgehen im Rahmen des Laborexperiments im Überblick.

Der Fragebogen wurde in mehreren Stufen erarbeitet (vgl. Abbildung 1). Zunächst waren je drei Aussagen zur eigenen Methodenkenntnis und zur zusammenfassenden Bewertung der jeweils zur Lösung der Planungsaufgabe angewendeten Methode gefragt. Zentral für die hier behandelte Fragestellung waren Aussagebatterien über die kommunikative, kreative und die rationale Dimension der Methode. Zudem wurde darin die Einschätzung der rationalen, kommunikativen und kreativen Dimension direkt abgefragt (,Die Methode ist vor allem rational/kommunikativ/ kreativ"). Der Fragebogen wurde von 19 Studierenden nach der Bearbeitung einer Planungsaufgabe mit der SWOT- 


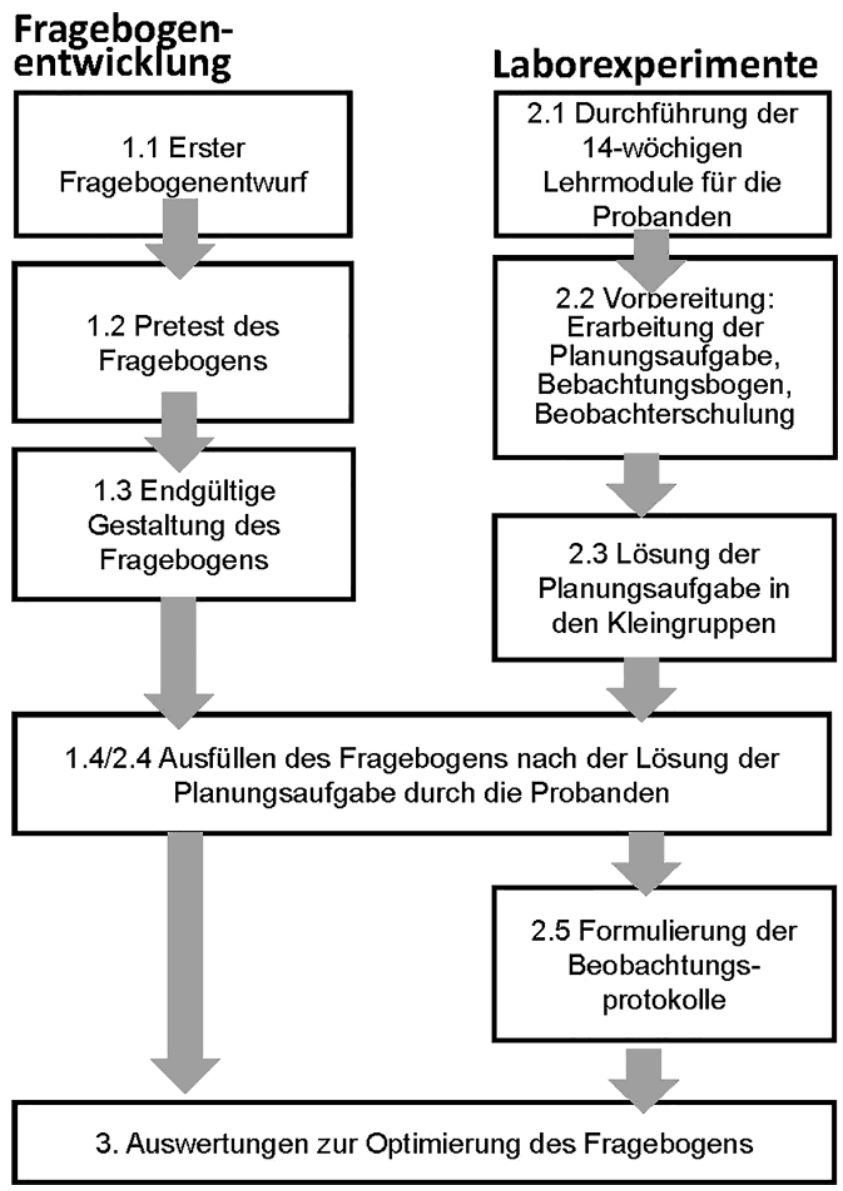

Abbildung 1 Vorgehen von der Entwicklung eines Fragebogens zur Messung der Bewertung der Dimensionen von Planungsmethoden im Rahmen eines laborexperimentellen Designs

Analyse und dem Mind Mapping erprobt. In mehreren Stufen wurde dann sowohl der Inhalt des Fragebogens erweitert als auch das Design optimiert. Grundlage für die Erweiterung des Fragenkatalogs waren einerseits weitere parallel durchgeführte Literaturrecherchen zur Beschreibung der Begriffe „rational“, „kommunikativ“, „kreativ“ und zur Fragebogenerstellung (z. B. Sternberg 2002; Jetter/Skrotzki 2005; Rost 2010; Winkler/König/Kleinmann 2010) und andererseits ein Brainstorming mit Studierenden zu den drei Dimensionen. In einem Pretest wurden dann die Aussagen für die Planungsmethoden Mind Mapping, SWOT-Analyse und Nutzwertanalyse bewertet. Möglich war eine Bewertung von 0 (trifft überhaupt nicht zu) bis 4 (trifft voll und ganz zu). Die Ergebnisse des Pretests verdeutlichen, dass eine Abgrenzung der gebildeten Dimensionen zueinander möglich ist, jedoch auch, dass sich die zu bewertenden Methoden hinsichtlich der Dimensionen voneinander unterscheiden.

Der wichtigste Aussagenkomplex innerhalb des endgültigen Fragebogens enthielt zu jeder Dimension zehn zu bewertende Aussagen, die im Fragebogen zufallsverteilt ange- ordnet waren, damit die Probanden die deduktiv abgeleitete Zuordnung zu den Dimensionen nicht erkennen konnten (vgl. Tabelle 1).

Der zweite Fragenkomplex des Fragebogens beinhaltete Aussagen zu Methodenvorkenntnissen, Methodenkompetenz und zur Nützlichkeit der Methoden. Im Einzelnen wurden dabei folgende Aspekte durch die nachfolgend aufgeführten Aussagen ermittelt:

- Verständlichkeit der Methode durch die Aussage „Die Methode war einfach zu verstehen.“

- Methodenvorkenntnisse durch die beiden Aussagen: „Die Methode kannte ich bereits vor der Sitzung.“ und „Ich habe die Methode bereits vor der Sitzung angewendet."

- Methodenkompetenz durch die drei Aussagen: „Ich kann anderen die Methode erklären.“, „Ich bin in der Lage, ein Planungsproblem mit dieser Methode zu lösen." und „Die Methode war einfach anzuwenden.“

- Nützlichkeit der Methode und Qualität der Informationsbereitstellung durch die fünf Aussagen: „Die Methode war zur Lösung der Aufgabe hilfreich.“, „Mit Hilfe der Methode konnte ein Ergebnis erzielt werden.“, „Als Mitarbeiter/Mitarbeiterin des Planungsamtes würde ich die angewandte Methode nutzen, um ähnliche Probleme zu lösen.“, „Mit den zur Verfügung gestellten Informationen bin ich zufrieden.“ und „Die Methode konnte mit Hilfe der gegebenen Informationen gut angewendet werden."

- Gesamtzufriedenheit mit der Aufgabenerfüllung durch die Aussage: „Ich habe das Gefühl, die Aufgabe mit meiner Gruppe gut gelöst zu haben."

- Lerneffekte durch Methodeneinsatz in der Aufgabe durch die drei Aussagen: „Die Bearbeitung der Aufgabe hat dazu beigetragen, dass ich die Methode besser verstehe.", „Durch die Bearbeitung der Aufgabe konnte ich meine Methodenkenntnis verbessern.“ und „Nach der Lösung der Aufgabe beherrschte ich die Methode im Vergleich zur Situation vorher besser.“

Die Experimente mit den Studierenden, die darin je eine Planungsaufgabe mit einer Planungsmethode lösen mussten, wurde zwar in ihrem ,natürlichen' Umfeld der Universität durchgeführt, wie es eigentlich für Feldexperimente charakteristisch ist. Jedoch war die Situation, in der die Planungsaufgaben absolviert wurden, so stark kontrolliert und aus studentischer Sicht untypisch, dass hier von einem Laborexperiment gesprochen werden kann.

Die Wissensgrundlagen für die Probanden zu den im Rahmen der Planungsaufgaben eingesetzten Planungsmethoden waren vor allem durch einsemestrige 14-wöchige Lehrveranstaltungen im Rahmen des Bachelor-Studienganges Geographie im Zeitraum Wintersemester 2013/2014 bis Wintersemester 2015/2016 gelegt worden. In diesen Lehrveranstaltungen wurden folgende Methoden erlernt: 
Tabelle 1 Fragebogenentwicklung: Aussagen zur Messung der Dimensionen der Planungsmethoden

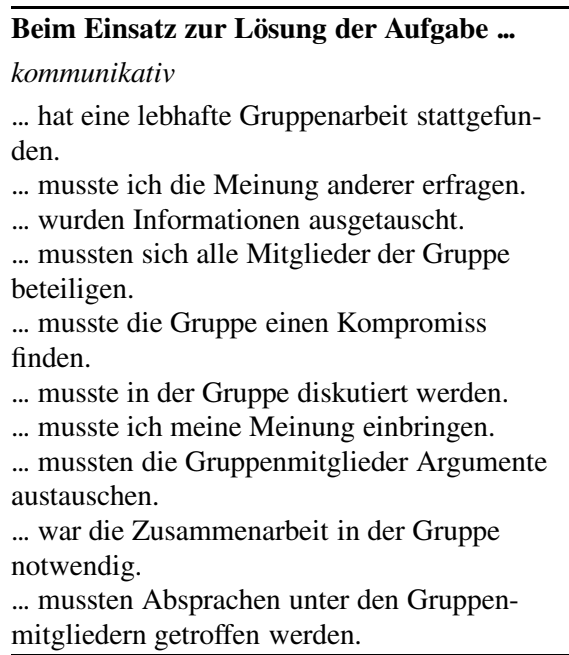

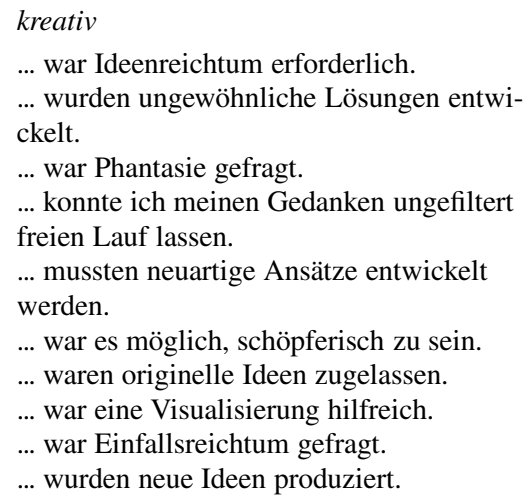

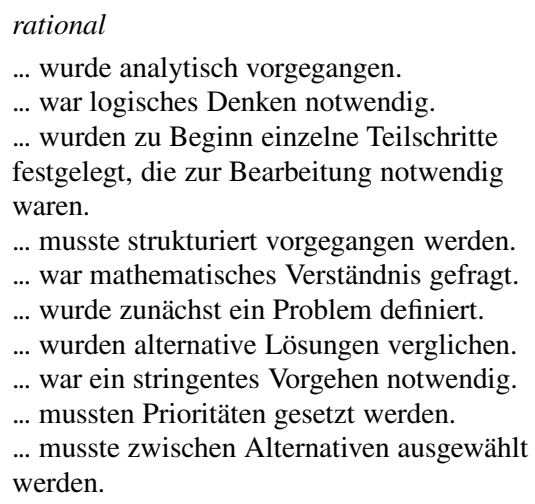

- Netzplantechnik, Planspiel, World Café, Mind Mapping im Rahmen des Moduls „Projektmanagement“

- SWOT-Analyse im Rahmen des „Einführungsprojekts Raumplanung“ und der Tutorien zur „Einführungsvorlesung Raumplanung“"

- Nutzwertanalyse im Rahmen des Moduls „Bewertungsmethoden“"

Im Rahmen der Module wurden die Methoden grundsätzlich erläutert und es wurden während des gesamten Verlaufs der Lehrveranstaltungen bereits kleine Übungen absolviert. Für diese Methoden konnte also am Semesterende von soliden Basiskenntnissen der Studierenden ausgegangen werden. Die Experimente bildeten den Abschluss der einsemestrigen Lehrveranstaltungen. Zusammen mit schriftlichen Ausarbeitungen ergaben sie auch einen Teil der Modulnote. Allerdings war, dies wurde vorher klar kommuniziert, die Teilnahme am Experiment verpflichtend, die Aktivitäten und Ergebnisse bildeten jedoch keinen Teil der Gesamtnote.

Darüber hinaus wurde ein Experiment mit der Methode des „Strategic Choice Approach“ (SCA) (vgl. Fuchs 2001; Friend/Hickling 2005; Diller/Oberding 2017) im Rahmen zweier Projektmodule der Masterklassen der Geographie an der Justus-Liebig-Universität Gießen in den Sommersemestern 2014 und 2015 durchgeführt. Der SCA ist eine komplexe Sammlung von Werkzeugen, mit deren Hilfe multidimensionale Entscheidungsprobleme gelöst werden können. Mit dieser Hilfe kann beispielsweise nicht nur, wie bei den konventionellen Bewertungsmethoden, über eine Verkehrstrassenvariante entschieden werden, sondern es können auch Entscheidungen über Standorte anderer Einrichtungen mit dieser Entscheidung verknüpft werden. Im Unterschied zu den anderen Experimenten erhielten hier die Studierenden nur eine kurze Einführung in die Metho- de. Sie mussten sich die Methode während der Anwendung im Experiment weiter erschließen. Im Gegensatz zu den anderen Gruppen fanden für diese Gruppe mehrere Sitzungen statt, die nicht beobachtet und von den Studierenden selbst geleitet wurden. Auch wenn es sich um Master-Studierende mit gewissen Grundkenntnissen zu anderen Methoden handelte, kann von deutlich weniger Grundkenntnissen als bei Praktikern der Raumplanung ausgegangen werden; die Studierenden wurden bewusst ,ins kalte Wasser geworfen ".

Im Zuge der Vorbereitung der Laborexperimente (vgl. Abbildung 1, AS 2.2). wurden von der Forschungsgruppe die im Experiment mithilfe der Planungsmethoden in Gruppenarbeit zu lösenden Planungsaufgaben erarbeitet und schließlich in einem Handout für die Studierenden fixiert. Die Aufgaben waren so gewählt, dass sie in Kleingruppen innerhalb der vorgegebenen Bearbeitungsdauer von $45 \mathrm{Mi}$ nuten absolviert werden konnten. Um dies sicherzustellen, wurden die Aufgaben von der Forschungsgruppe selbst getestet.

Das Laborexperiment selbst wurde im Zeitfenster der jeweiligen Lehrveranstaltungen (Ausnahme: Gruppe zum Strategic Choice Approach) durchgeführt, in der Regel am Abschlusstag, zum Teil aber auch an mehreren Tagen davor und danach. Für die Studierenden bestand Anwesenheitspflicht. Am Anfang wurde das Handout zur Planungsaufgabe an die Studierenden verteilt und die Planungsaufgabe wurde ausführlich erläutert. Die Zuteilung zu den Kleingruppen konnten die Studierenden selbst vornehmen, zum Teil entsprechend ihrer Methodenpräferenzen, in jedem Fall gemäß ihren Präferenzen zur Zusammenarbeit mit anderen Akteuren. Da somit die Stichproben (Arbeitsgruppen) nicht rein zufällig zusammengesetzt wurden, handelt es sich streng genommen nicht um ein Experiment, sondern ein Quasi-Experiment. Der daraus möglicherweise resultierende Nachteil der geringeren internen Validität von Quasi-Ex- 
Bei der Anwendung der Methode zur Lösung der Planungsaufgabe ..." (Skalenwert $0=$ trifft überhaupt nicht zu; Skalenwert $4=$ trifft voll und ganz zu)

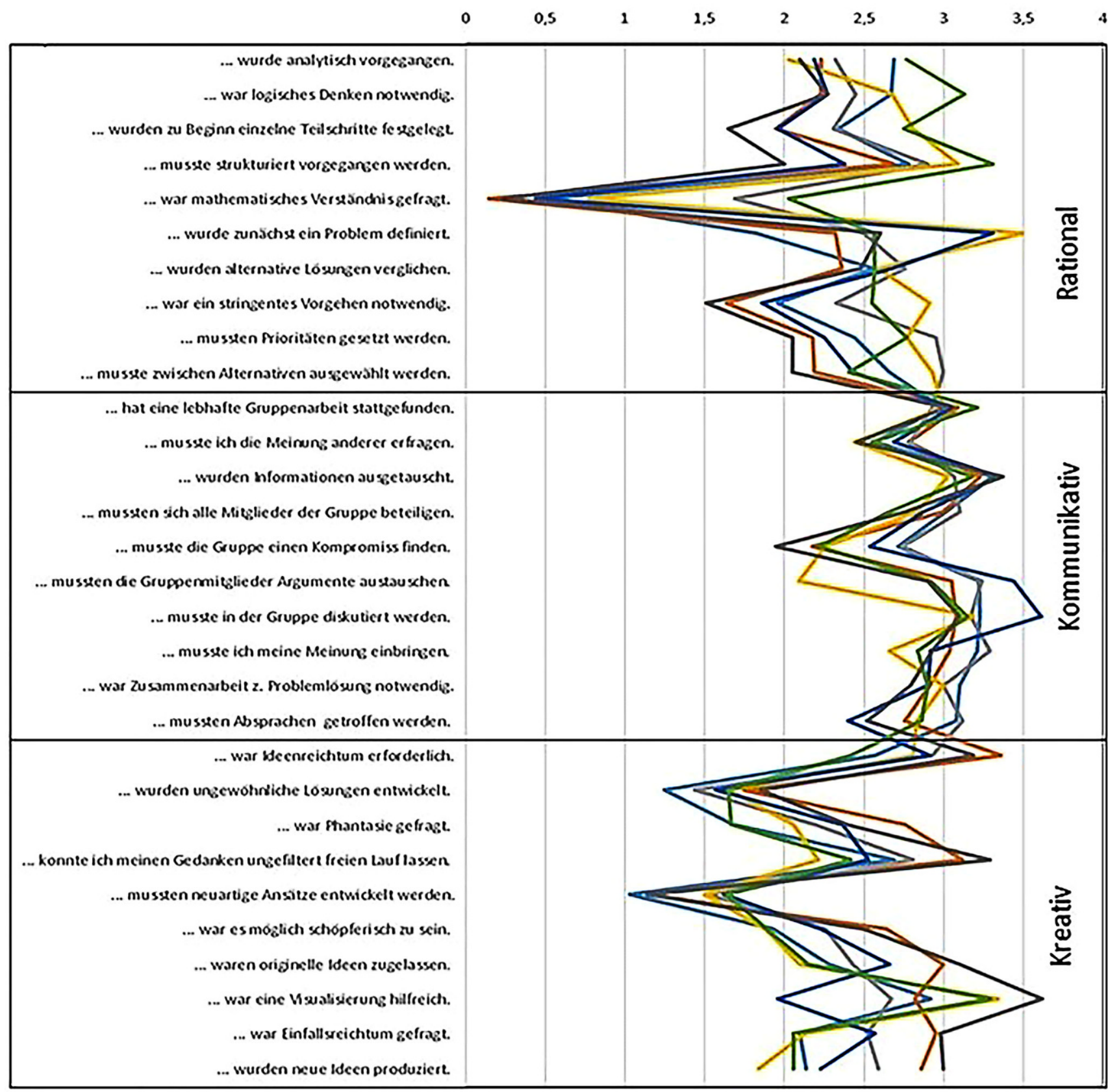

$\longrightarrow$ SVT.Analyse - Mind Mapping - Nutzwertanalyse —Strategic.Choice-Approach — Planspiel — Netzplantechnik -World Cafe

Abbildung 2 Einstufung von sieben Planungsmethoden mit einzelnen Aussagen (Items)

perimenten (vgl. Bortz/Döring 2016: 193) im Vergleich zu zufallsauswahlbasierten Experimenten wurde in Kauf genommen, um ein größeres Basiswissensniveau (die Studierenden wählten eher die Methoden, die sie beherrschten), eine reibungslosere Zusammenarbeit der Gruppe (die Studierenden arbeiteten in der Regel eher mit den ihnen gut bekannten Kommilitonen zusammen) und damit ein insge- samt höheres Niveau der Aufgabenerfüllung sicherzustellen und die Motivation der Probanden zu erhöhen.

In den Lehrveranstaltungen zur SWOT-Analyse und zur Nutzwertanalyse hatten die Studierenden keine freie Methodenwahl. In der Lehrveranstaltung „Projektmanagement“ war ihnen allerdings eine Methode aus dem Werkzeug-Katalog (World Café, Planspiel, Mind Mapping und Netzplan- 
technik) vorgegeben, die sie durch eine weitere ergänzen konnten, zu der dann ein eigener Bewertungsbogen ausgefüllt wurde. Die Beobachter waren in Hörweite zu den Probanden platziert und standen grundsätzlich während der gesamten Bearbeitung für Rückfragen zur Verfügung. Auf das Einhalten der vorgegebenen Bearbeitungszeit von 45 Minuten wurde geachtet. Nach der Bearbeitung der Aufgabe erhielten die Probanden den oben beschriebenen Fragebogen, in dem die Methode, die zur Lösung der Aufgabe eingesetzt wurde, bewertet werden sollte. Das Ausfüllen war Pflicht, danach wurde der Bogen eingesammelt.

Insgesamt wurden die Laborexperimente während der Sommersemester 2014 und 2015 an 37 Terminen (je Termin eine Stichprobe) in Kleingruppenarbeit durchgeführt. Die Teilnehmerzahl je Gruppe lag zwischen drei und sieben. Insgesamt wurden nach den Experimenten zu den sieben Planungsmethoden 518 gültige Fragebögen (Fälle) abgegeben: Netzplantechnik (160 Antworten), SWOT-Analyse (139), Planspiel (77), Nutzwertanalyse (42), World Café (42), Strategic Choice Approach (32), Mind Mapping (26). Die durchschnittliche Stichprobengröße lag bei 14 .

\subsubsection{Ergebnisse}

Die Auswertung der durchgeführten Untersuchungen erbrachte interessante Ergebnisse zu Fragen der Nützlichkeit der einzelnen Planungsmethoden. Diese sind jedoch an anderer Stelle dokumentiert (Diller/Karic/Oberding 2017). Die folgende Darstellung bleibt auf den Aspekt der in der Hypothese aufgeführten drei Bewertungsdimensionen fokussiert.

Abbildung 2 zeigt zunächst die Aussagen für die einzelnen Methoden. In der Darstellung sind die Antworten jetzt anders als im Fragebogen sortiert: im oberen Drittel die Aussagen der, rationalen ' Dimension, im mittleren Drittel die Aussagen der ,kommunikativen ' Dimension und im unteren Drittel die Aussagen der ,kreativen“ Dimension. Je höher der Skalenwert, desto größer die Zustimmung zu der Einschätzung.

Folgende Ergebnisse lassen sich finden: Bei der Aussagengruppe der ,rationalen ' Dimension sind die Unterschiede zwischen den Methoden relativ auffällig. Die größten Unterschiede zeigen sich bei der Aussage „Bei der Anwendung der Methode war mathematisches Verständnis gefragt.". Lediglich für die Nutzwertanalyse und die Netzplantechnik wird die Aussage bejaht. Aber auch die Aussagen ,... musste strukturiert vorgegangen werden“ und ,,... war ein stringentes Vorgehen erforderlich" werden für die Methoden recht unterschiedlich beantwortet. Netzplantechnik und Strategic Choice Approach sowie die Nutzwertanalyse erhalten bei diesen Aussagen die höchsten Zustimmungswerte. Bei den Aussagen, die die ,kommunikative Dimension betreffen, sind die Antwortpunkte zum einen bei allen Methoden insgesamt relativ hoch und sind zum anderen die Unterschiede zwischen den Methoden insgesamt geringer als bei den anderen Dimensionen. Lediglich bei den Items ,,... mussten Kompromisse gefunden werden“ und ,... mussten die Gruppenmitglieder Argumente austauschen" öffnet sich die Schere zwischen den Methoden etwas weiter. SWOT-Analyse und Nutzwertanalyse liegen in dieser Fragengruppe relativ weit vorne, Planspiel und World Café punktuell ebenfalls, aber weniger deutlich als für diese kommunikativen Formate zu erwarten war. Bei der ,kreativen' Dimension werden die einzelnen Aussagen sehr unterschiedlich eingeschätzt: ,,... war Ideenreichtum erforderlich“ und ,... konnte ich meinen Gedanken freien Lauf lassen“ werden besonders oft genannt, die Aussagen „wurden ungewöhnliche Lösungen entwickelt“ und ,... mussten neuartige Ansätze entwickelt werden" relativ selten. World Café und Mind Mapping liegen - nicht überraschend - bei den Fragen aus dieser Gruppe in der Bewertung vorne.

Abbildung 3 zeigt die aus den Aussagen aggregierten Ergebnisse bezogen auf die drei Dimensionen ,kommunikativ', ,kreativ' und ,rational'. Dargestellt ist der jeweilige Anteil an allen aufsummierten Mittelwerten der Antworten zu den je zehn Aussagen in den drei Dimensionen.

Aus dieser Abbildung werden vor allem zwei Ergebnisse deutlich: Zum einen variieren die aggregierten Punktwerte für die einzelnen Dimensionen bei den Methoden zwar hochsignifikant mit mittlerer Korrelation (Eta: 0,3$0,4)$ bei der ,rationalen “ und ,kreativen“ Dimension oder zumindest einfach signifikant mit geringen Korrelationen (Eta um 0,2) bei der ,kommunikativen ' Dimension. Dennoch kann keine Methode eindeutig einer Dimension zugeordnet werden. Zum anderen liegen bei allen Methoden die Zustimmungspunktwerte zu den Aussagen auf der ,kommunikativen ' Dimension am höchsten, aber auch hier sind die Unterschiede gering. Die Methoden unterscheiden sich eher darin, ob die ,rationale“ (Netzplantechnik, Nutzwertanalyse,

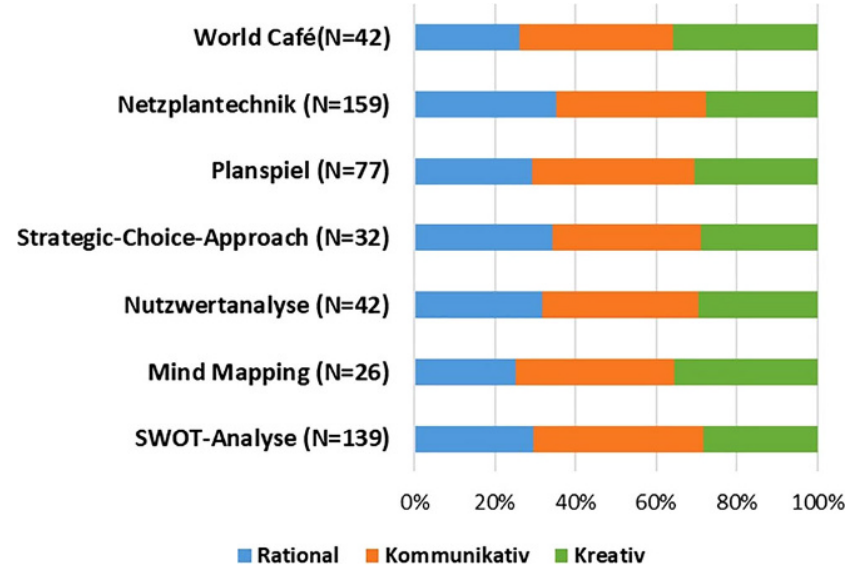

Abbildung 3 Einstufung von sieben Planungsmethoden: Zusammenfassung zu drei Bewertungsdimensionen (aggregierte Werte) 
Abbildung 4 Einstufung von sieben Planungsmethoden: $\mathrm{Zu}$ sammenfassende Bewertung seitens der Probanden

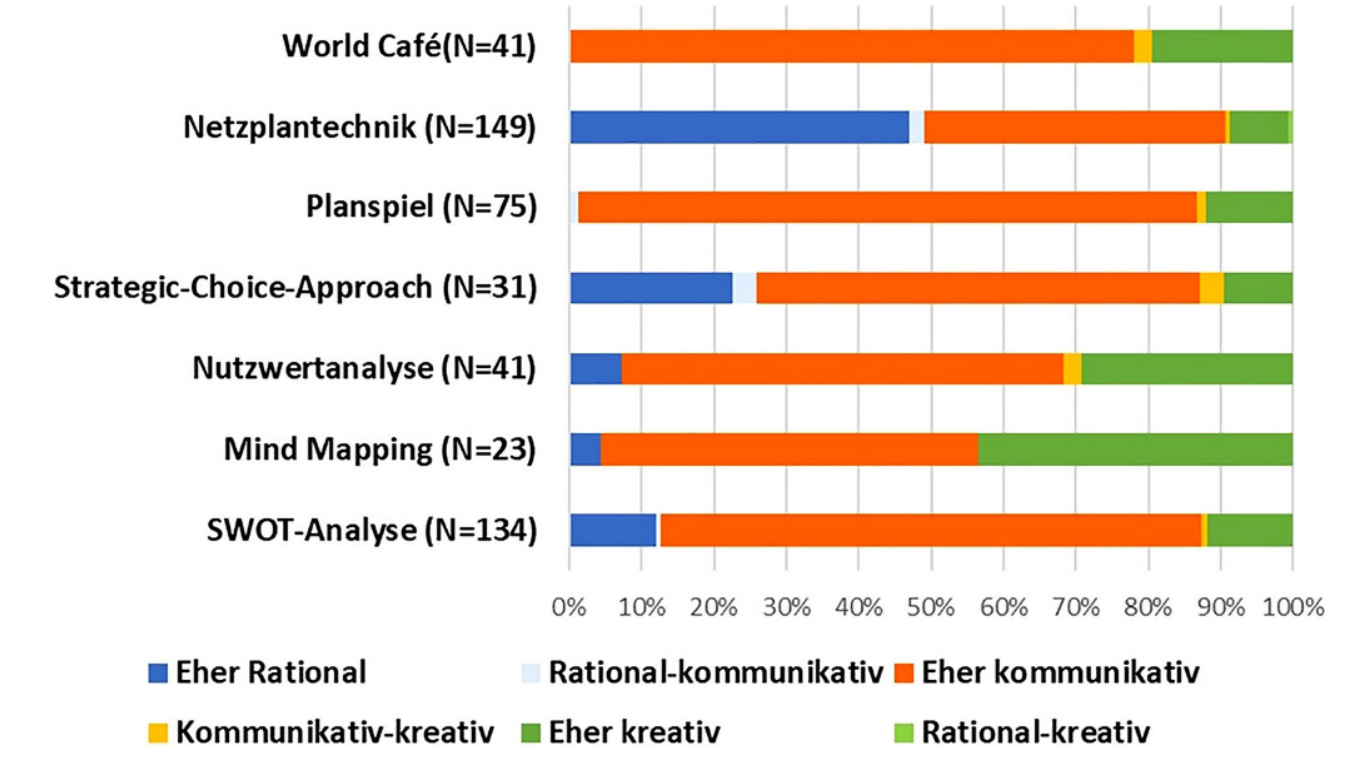

World Café( $\mathrm{N}=41)$

Netzplantechnik ( $\mathrm{N}=149)$

Planspiel $(\mathrm{N}=75)$

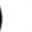
)

Mind Mapping ( $\mathrm{N}=23)$

SWOT-Analyse $(\mathrm{N}=134)$

Rational-kommunikativ a Eher kommunikativ

Kommunikativ-kreativ

Strategic Choice Approach) oder die ,kreative“ Dimension tig von ,kommunikativen Methoden ', ,rationalen Methoden “ oder ,kreativen Methoden' sprechen zu können. Wie kommunikativ eine Methode von Akteuren, im Falle dieser Untersuchung von Studierenden, empfunden wird, hängt also nicht nur von der Methode selbst, sondern vor allem von dem Setting (verstanden als situativer Rahmen des Methodeneinsatzes mit spezifischen Orten und zeitlichen Rahmen), in dem sie angewandt wird, ab. Auch klassische analytisch-rationale Methoden können mal mehr oder weniger kommunikativ ausgestaltet werden.

Damit stellt sich die Frage, ob sich der „Communicative Turn" in der Planungstheorie auch in der Weiterentwicklung einzelner Methoden zeigt und ob und wie klassische rationale Methoden entsprechend kommunikativ weiterentwickelt werden können. Dies wird nachfolgend anhand einer multiattributiven Bewertungsmethode diskutiert: der Nutzwertanalyse.

zwei Methoden, die die Mehrzahl der Probanden zusammenfassend als ,eher kommunikativ“ einstuften: die Netzplantechnik, die noch ein wenig mehr als ,eher rational" gilt, und das Mind Mapping, das in fast ebensolchem Maße als ,eher kreativ“ wie ,eher kommunikativ“ gilt.

\subsection{Fazit der empirischen Untersuchung}

Das zentrale inhaltliche Ergebnis der Experimente und der Befragungsauswertung bezogen auf die Ausgangshypothesen zu den Methoden (vgl. Kapitel 2.1 und Abbildung 1) ist, dass es keine Methode gibt, die eindeutig als ,rational', ,kommunikativ' oder ,kreativ' eingeordnet werden kann. Die ermittelten Gewichte der ,rationalen', ,kommunikativen" und ,kreativen" Dimension der Methoden variieren zwar, aber die Unterschiede sind zu gering, um einzelne Methoden klar einer Dimension zuzuordnen, also eindeu-

\section{Kommunikative Weiterentwicklungen rationaler Planungsmethoden am Beispiel der Nutzwertanalyse}

Die Nutzwertanalyse wurde in ihrer ,ersten Generation“ in Deutschland von Zangemeister (1970) erstmals systematisch vorgestellt. Sie setzt vor allem an einem Mangel der bis dahin (und auch heute noch) in der Planung für große Infrastrukturprojekte wichtigsten Methode, der Nutzen-Kosten-Analyse, an: der Tatsache, dass Nutzen nur mit großem Aufwand und oftmals nicht wissenschaftlich-seriös in Geldwerten ausgedrückt werden können. Die Nutzwertanalyse stellt daher nicht die Effizienz als monetär ausdrückbare Kosten-Nutzen-Relation, sondern die Effektivität 
in das Zentrum der Betrachtung. In ihrer klaren Grundstruktur bildet die Nutzwertanalyse das klassische lineare Modell des Planungsprozesses ab. Zunächst wird in einem ersten Bewertungsvorgang ein hierarchisches Zielsystem mit festen Zielgewichten entworfen. Vor dem Hintergrund dieses festen Zielsystems werden in einem zweiten Bewertungsvorgang für die zu bewertenden Alternativen zielbezogene Erreichungsgrade mit Nutzenfunktionen zu Gesamtnutzen addiert. Die formale Transparenz der Nutzwertanalyse erklärt sich auch durch die klare Trennung der beiden Bewertungsvorgänge. Der erste Bewertungsvorgang (Zielgewichtung) ist vor allem subjektiv wertebasiert (substanzielle Rationalität), der zweite (Zielerreichungsgradermittlung) ist stärker am Maßstab objektiver Wissenschaftlichkeit (objektive Formalrationalität) orientiert und setzt vor allem Expertensachverstand beim Abschätzen möglicher Wirkungen voraus. Politische Entscheidungsträger und Bürger sind vor allem für die erste Bewertungsstufe maßgeblich, Experten für die zweite. Die Verbindung zwischen den beiden Bewertungsvorgängen ist letztlich durch schematische mathematische Operationen gewährleistet. Die Differenzierungen der Methode bestehen vor allem in mathematisch nachvollziehbaren Entscheidungsregeln für die Wertsynthesen.

Die Nutzwertanalyse der „ersten Generation“ war die Planungsmethode, die das klassische rationale Planungsmodell am besten widerspiegelt. Insofern waren auch die an ihr geübten Kritikpunkte in weiten Teilen deckungsgleich mit den Kritikpunkten am klassischen rationalen Planungsmodell: Der lineare Ablauf vernachlässige Rückkopplungen und Lernprozesse, das kardinale Messniveau suggeriere eine letztlich nicht vorhandene Objektivität und Genauigkeit der Bewertung. Die nötigen stetigen Nutzenfunktionen existieren für die meisten Indikatoren gar nicht. Zudem führe die Aggregation der Teilnutzen zum Gesamtnutzen da$\mathrm{zu}$, dass die Nutzwertanalyse als intransparente Black Box eingesetzt werde und Tabukriterien nicht dezidiert als solche behandelt werden können. Die unterstellte Ziel- und Nutzenunabhängigkeit und Substituierbarkeit der einzelnen Kriterien entspräche nicht dem alltäglichen Vorgang, mit dem Menschen bewerten (Fürst/Scholles 2008b; Nollau/ Gottfried 2009: 116).

Nicht alle diese Kritikpunkte sind gerechtfertigt. Beispielsweise wird bei der Kritik häufig vergessen, dass ein Element der Nutzwertanalyse der ,ersten Generation" durchaus bereits der Anforderung der Reflexivität und Reversibilität entspricht, nämlich die Sensititivätsanalyse. Diese sieht vor, dass im letzten Schritt der erste Bewertungsschritt der Zielgewichtungen überprüft und gegebenenfalls revidiert werden kann (Pommer 2007: 75f.). Insofern ist die Nutzwertanalyse trotz klarer Abfolge der Schritte keine starre, nur linear angelegte Methode. Betrachtet man die Weiterentwicklungen der Nutzwertanalyse und später vorgeschlagene alternative Bewertungsmethoden aus der Gruppe multikriterieller Entscheidungsverfahren (Pommer 2007: 68ff.), so setzen die meisten von diesen an den Kritikpunkten der Nutzwertanalyse an und versuchen, diese so weiterzuentwickeln, dass sie dem natürlichen Bewertungsvorgehen des Menschen eher entspricht.

Die in der zweiten Hälfte der 1970er-Jahre in Deutschland von Bechmann (1978) (zur Anwendung vgl. Bechmann/Kiemstedt 1974) vorgestellte Nutzwertanalyse der „zweiten Generation“ setzt an Schwachstellen der Nutzwertanalyse der „ersten Generation“ an, erstens an der Frage nach der Eignung der Urteilspersonen für die beiden Bewertungsstufen, zweitens an der Annahme der Nutzenunabhängigkeit der einzelnen Kriterien, drittens am unterstellten kardinalen Messniveau, das die Wertsynthese der Zusammenfassung der Teilnutzen zu einem Gesamtnutzen ermöglicht. Der entscheidende Unterschied zur Nutzwertanalyse der „ersten Generation“ ist der Rückgang auf das ordinale Messniveau und die Flexibilisierung des Bewertungsvorganges. Die Bewertung wird nicht auf der Basis fester Zielgewichte für jedes einzelne Kriterium vorgenommen, sondern es können jeweils mehrere Kriterien gleichzeitig betrachtet und in der Zusammenschau flexibler amalgamiert werden. Damit wird dem Umstand Rechnung getragen, dass Bewertungen eines Ziels auch von der Bewertung eines anderen Ziels abhängen. Die Wertsynthese erfolgt nicht durch kardinale Aggregation, sondern ist von inhaltlichen Überlegungen geleitet und wird durch logische Verknüpfungen unter Einsatz der Boolschen Algebra ${ }^{2}$ vorgenommen, womit das kardinale Messniveau verlassen wird. Die beiden getrennten Bewertungsvorgänge der Nutzwertanalyse der „ersten Generation“ (Zielgewichtung und Ermittlung der Zielerträge) werden hier zu einem Vorgang fusioniert. Der Nachteil ist, dass die Verarbeitungskapazitäten im Hinblick auf die Kriterienzahl im Vergleich zur Nutzwertanalyse der ,ersten Generation“ reduziert sind und die Bewertungsvorgänge, da sie fusioniert werden, nach außen weniger transparent sind (Fürst/Scholles 2008b: 450). Zudem ist mit dem Absenken des Messniveaus zumindest mathematisch ein Informationsverlust verbunden. Dennoch hat die Nutzwertanalyse zumindest indirekt die Methoden der Umweltverträglichkeitsprüfungen, insbesondere die dort verbreitete Risikoanalyse, maßgeblich inspiriert.

Der ebenfalls in der zweiten Hälfte der 1970er-Jahren von Saaty (1977) vorgestellte „Analytic Hierarchy Process" (Analytischer Hierarchieprozess; AHP) unterscheidet sind von der Nutzwertanalyse der ,ersten Generation“ vor allem darin, dass die Zielgewichtungen schrittweise durch den paarweisen Vergleich einzelner Ziele aus dem hierarchischen Zielsystem erarbeitet werden. Der Vorgang

\footnotetext{
${ }^{2}$ Unter der Boolschen Algebra wird eine spezielle Struktur verstanden, die unter anderem die Eigenschaften der Operatoren UND, ODER, NICHT verallgemeinert.
} 
ist aufwendiger, da jedes Ziel explizit mit jedem anderen verglichen wird; die Anforderungen an die Präferenzartikulation des Entscheidungsträgers erscheinen höher als bei der Nutzwertanalyse der „ersten Generation“ (Beck 2011: 14). Wegen neuer Möglichkeiten der Datenverarbeitung (vgl. Kou/Ergu/Peng et al. 2013) tritt dieses Problem allerdings zunehmend in den Hintergrund und der Vorteil des Analytischen Hierarchieprozesses kommt zum Tragen: Seine Bewertungsergebnisse erscheinen durch den schrittweisen Paarvergleich der Ziele robuster (Siebert 2009: $22 \mathrm{ff}$.). Das dürfte der Grund sein, warum diese Methode international stärker verbreitet ist als die in Deutschland relativ bekannte Nutzwertanalyse (Pommer 2007: 77). Eine Weiterentwicklung des Analytischen Hierarchieprozesses stellt der analytische Netzwerkprozess dar, der auch die Möglichkeit der wechselseitigen Abhängigkeit der Ziele auf unterschiedlichen Hierarchieebenen ermöglicht (Pommer 2007: 105 ff.). Auch bei der Multiattributiven Nutzentheorie (MAUT) werden Teilnutzen durch direkten paarweisen Vergleich von Zielbeiträgen gleichen Nutzens ermittelt (Schäfer/Witte 2014: 90).

Diese beiden prominentesten Weiterentwicklungen bzw. Alternativen zur Nutzwertanalyse unterscheiden sich von dieser also vor allem darin, dass mit ihnen versucht wird, der beschränkten Rationalität menschlichen Bewertungsvermögens durch einen noch flexibleren und durch direkte Paarvergleiche einfacheren Bewertungsvorgang entgegenzukommen. Auch andere und neuere Weiterentwicklungen der Nutzwertanalyse versuchen, etwa durch Einführungen von Fuzzy-Logiken (Rommelfanger/Eickemeier 2002: 173) oder der Vektorrechnung, (Nollau/Gottfried 2009) Schwächen der Nutzwertanalyse der „ersten Generation“ abzumildern und mathematische Logik mit realem menschlichem Denken besser in Einklang zu bringen.

Trotz der geäußerten Kritik findet die Nutzwertanalyse der ,ersten Generation“ weiterhin in vielen Disziplinen Anwendung. Obwohl auch in der Raumplanung neue Themen, wie etwa die Klimaforschung, immer wieder Impulse für methodische Anleitungen liefern (Kersting/Werbeck 2014), scheint die Methode in den Planungswissenschaften weitaus weniger prominent als beispielsweise in der Betriebswirtschaftslehre, wo sie zum Teil unter anderen Begriffen wie „Scoring-Modell“ firmierte (Weber 2004) und aus ihr auch ähnliche Methoden wie die Balanced Scorecard entwickelt wurden (Vohl 2004). Trotz ihrer Modifikationen bleibt die Nutzwertanalyse eine Methode, die dem klassischen rationalen Planungsmodell folgt.

Wie stellte sich die Nutzwertanalyse im Rahmen der in Kapitel 2 dargestellten Experimente dar? Die Abbildungen 2, 3 und 4 verdeutlichen, dass die Nutzwertanalyse zumindest innerhalb des gewählten Settings von den Probanden als Methode eingeschätzt wird, in der die kommunikative Dimension, wie bei allen Methoden, die stärkste
Ausprägung enthält. Dabei überrascht vor allem das Ergebnis der prägnanten zusammenfassenden Bewertung der Probanden (vgl. Abbildung 4). Hier wird nicht nur die kommunikative, sondern auch die kreative Dimension der Nutzwertanalyse deutlich höher eingeschätzt als die rationale.

Der Blick auf die Bewertung der Nutzwertanalyse (vgl. Abbildung 2) macht zunächst - nicht überraschend - deutlich, dass sie bei den Aussagen ,... mussten Prioritäten gesetzt werden“, ,,... wurden Alternativen bewertet", und ,,... musste zwischen Alternativen ausgewählt werden" höher als die anderen Methoden bewertet wird, denn sie ist ja dezidiert als Methode zur Alternativbewertung konzipiert. Überraschender ist, dass die Nutzwertanalyse auch in den meisten Fragen zu den kommunikativen Aspekten relativ hohe Werte, auch im Vergleich zu den anderen Methoden, erhält. Sie erscheint als Methode zur Anwendung in Gruppen sehr gut geeignet. Und auch in der Aussagegruppe „kreativ" fällt die Nutzwertanalyse lediglich bei den Fragen innovativer Lösungen etwas gegenüber den anderen Methoden $a b$.

Die oben beschriebenen Experimente machen deutlich, dass es vor allem vom Setting abhängt, wie kommunikativ eine Nutzwertanalyse oder andere Bewertungsmethoden angelegt sind. Zwar ist die Nutzwertanalyse in Lehrbüchern und an Anwendungsbeispielen vielerorts diskutiert, jedoch liegen nur wenige Arbeiten vor, die explizit die Anwendung der Nutzwertanalyse durch Akteure unter dem Aspekt der Partizipation zum Gegenstand haben: Zampich (2002) nimmt eine Bewertung von Eignungsflächen für Windenergieanlagen einer Gemeinde im Regierungsbezirk Münster mithilfe einer Nutzwertanalyse der „ersten Generation“ ”or. Dabei werden vier Standorte nach elf Kriterien bewertet. Während der Autor den Zielerreichungsgrad der Varianten selbst erhebt, werden die Zielgewichte durch insgesamt fünf befragte Akteure unterschiedlicher Interessengruppen ermittelt. Insofern ist die Nutzwertanalyse zumindest ansatzweise partizipativ angelegt. Trotz unterschiedlicher Präferenzen der Akteure bei der Zielgewichtung werden letztlich die Standortvorschläge eines vorher erstellten Expertengutachtens bestätigt.

Für eine Bewertung von Kultureinrichtungen in der Stadt Leipzig wurde der Einsatz einer Nutzwertanalyse in einem kommunikativen Prozess durchgeführt (Lenk/Oelsner/ Reimann 2010). Unter der Federführung des Kulturdezernats der Stadt, unterstützt durch einen externen Berater, entwarf der siebenköpfige Kulturbeirat in einem intensiven Diskussionsprozess zunächst ein gewichtetes Zielsystem der kommunalen Politik. Mithilfe eines aus elf Kriterien bestehenden Zielsystems wurden dann 17 kulturelle Einrichtungen der Stadt bewertet. Der Entwurf zur Bewertungsliste gelangte durch eine Indiskretion an die Presse und wurde dort als Streichliste dargestellt, weswegen vom ursprünglichen Ziel einer Klassifizierung Abstand genom- 
men werden musste. In der Bewertung wurden die Vorteile der Methode, multiple Ziele einzubeziehen und auch nichtmonetäre Kriterien einfließen zu lassen, positiv hervorgehoben. Entscheidend dafür, dass das verfolgte Ziel eines abgestimmten Rankings nicht eingehalten wurde, waren aber die Faktoren der Prozessgestaltung. Zum einen wurden die betroffenen Kultureinrichtungen nicht frühzeitig einbezogen, zum andern gelangten die Ergebnisse vorzeitig an die Presse. In der Bewertung des Prozesses räumen die Autoren somit ein, dass eine isolierte Nutzwertanalyse kein ausreichendes Instrument für kulturpolitische Entscheidungen darstellt. Sie betonen aber den Prozessnutzen. Die eingesetzte Methode führe zu einer Versachlichung der Diskussionen und dazu, dass reine Lobbyistenargumentationen gegenüber Gesamtnutzenargumenten an Gewicht verloren und die Argumente insgesamt transparenter wurden. Aufgrund dieser verfahrensstrukturierenden Funktion der Nutzwertanalyse fällt die Bilanz des Einsatzes dieser Methode dennoch positiv aus.

Beck (2011) führt unter der Prämisse, dass Partizipation eine Verbesserung der Bewertungsmethoden bringt, eine vergleichende Studie der Anwendung von Nutzwertanalyse und dem Analytischen Hierarchieprozess zur Lösung von Problemstellungen aus der Waldwirtschaft mit einem breiten Akteurkreis durch. Als Ergebnis der quasi-experimentellen vier Fallstudien zeigen sich Auswirkungen der Methodik. Beim Analytischen Hierarchieprozess sind die Unterschiede der Alternativen größer als bei der Nutzwertanalyse. Aus den Interviews mit den anwendenden Akteuren wird deutlich, dass die den Methoden innewohnende Trennung von Sach- und Wertebene positiv als Beitrag zur Versachlichung der Diskussion geschätzt wird. Der Analytische Hierarchieprozess gilt insgesamt als vollständiger und kleinteiliger strukturiert und reflexiver, was von den Stakeholdern positiv bewertet wird. Die Nutzwertanalyse gilt als etwas einfacher und manipulationsanfälliger. Die Methoden werden zwar zum Teil aufgrund der unverständlichen Fachbegriffe kritisiert, jedoch wird ihr Beitrag zur Verfahrensstrukturierung und Transparenzerhöhung deutlich positiv bewertet.

Vor dem Hintergrund des „Communicative Turn“ in der Raumplanung(stheorie) verwundert es weiterhin, dass es in den Lehrbüchern der Raumplanung kaum systematische, explizite Überlegungen zur partizipativen Anwendung von Bewertungsmethoden wie der Nutzwertanalyse gibt. Dabei ist doch gerade die Nutzwertanalyse der „ersten Generation" eine Methode mit hoher Eignung für Kollektiventscheidungen. Der Bewertungsprozess der Zielgewichte kann grundsätzlich sogar durch einen mengenmäßig nahe$\mathrm{zu}$ unbegrenzt breiten Akteurkreis als quasi-plebiszitäres Element erfolgen. Der Schritt der Zielertragsermittlung setzt zwar Expertenwissen voraus, kann aber dennoch in diskursiven Prozessen vollzogen werden. Die Stufe der
Sensitivitätsanalyse ist in hohem Maße ein reflexives Element, bei dem nach Kenntnis des Bewertungsergebnisses auch die zugrunde gelegten Ziele wieder überprüft und revidiert werden können. In manchen Lehrbüchern aus der Entscheidungstheorie der Betriebswirtschaft finden sich immerhin einige grundsätzliche Überlegungen, mit welchen Methoden bei der Anwendung individuelle zu kollektiven Entscheidungen zusammengeführt werden können (z. B Bildung gewogener oder ungewogener Durchschnitte, schrittweise Angleichung der Urteile durch Delphi-Methoden und Diskussionen, die bei Nichtkonsens durch Abstimmungen entschieden werden; vgl. Pommer 2007: $105 \mathrm{ff}$; Schäfer/Witte 2014: 76) und welche Entscheidungsregeln dabei angewendet werden können (z.B. Einstimmigkeitskriterium, Mehrheitsentscheidungen unter Umständen in mehreren Stufen, Borda-Kriterium, Condorcet-Alternative; vgl. Rommelfanger/Eickemeier 2002: $191 \mathrm{ff}$.).

\section{Fazit und weiterer Forschungsbedarf}

Im ersten Kapitel wurde die vor allem in den 1970er-und 1980er-Jahren in der Raumplanungsforschung, aber auch anderen Disziplinen intensiv geführte Rationalitätsdebatte nachgezeichnet. Die von Siebel (2006) vorgeschlagene pragmatische Synthese der Modelle der beiden konträren Rationalitätsbegriffe, der klassischen Zweck-Mittel-Rationalität und der kommunikativen Rationalität, erscheint dem Planungsalltag und damit auch der Untersuchung von Planungsmethoden angemessen. Bezogen auf den Untersuchungsgegenstand von Planungsmethoden bedarf sie allerdings der Ergänzung durch die Dimension der emotionalen Rationalität (Schubert 2014), da diese vermutlich eine wichtige Dimension bei den relevanten Kreativitätsmethoden (die nicht per se kommunikativ ausgelegt sein müssen) darstellt. Die Synthese der Modelle lässt sich letztlich auf die Formel zuspitzen, dass es vor allem die beiden Rationalitätsbegriffe der klassischen analytischen Rationalität einerseits und der kommunikativen Rationalität andererseits sowie die stark durch emotionale Elemente geprägte Kreativität sind, mit denen raumplanerische Problemstellungen angegangen werden und die situationsbedingt in unterschiedlichen Gewichtungen zum Tragen kommen.

Im zweiten Kapitel wurde dann der Leitfrage des Beitrags nachgegangen, ob die unterschiedlichen Rationalitätsbegriffe sich auch in den eingesetzten Planungsmethoden zeigen. Das in dieser Deutlichkeit überraschende Ergebnis aus den im Rahmen eines Forschungsvorhabens durchgeführten (Quasi-)Laborexperimenten mit geschulten studentischen Probanden war, dass die Trennung zwischen ,rationalen " und ,kommunikativen' Methoden - als weitere Bewertungsdimension wurde die ,kreative" einbezogen bestenfalls eine graduelle ist. Methoden sind also nicht ,ra- 
tional', ,kommunikativ“ und ,kreativ' an sich, es kommt auf das Rahmen des Einsatzes an.

Dies bedeutet, dass an die Stelle der Ausgangshypothese H1 Planungsmethoden lassen sich in ihren Bedeutungsanteilen der rationalen, kommunikativen und kreativen Dimension und damit in ihren Möglichkeiten, die entsprechenden Potenziale der Akteure in Planungsprozessen zu aktivieren, eindeutig unterscheiden nunmehr die Endhypothese Settings in Planungsprozessen, in denen Planungsmethoden eingesetzt werden, unterscheiden sich graduell im Hinblick auf die Bedeutungsanteile der rationalen, kommunikativen und kreativen Dimension und damit in ihren Möglichkeiten, Potenziale von Akteuren zu aktivieren tritt. Von ,rationalen“ vs. ,kommunikativen" Methoden kann also im Sinne einer prägnanten Zuspitzung gesprochen werden. Insofern bestätigen sich für die Hypothese 2 die für die Bedeutungsdimensionen formulierten Anführungszeichen.

Daraus ergibt sich auch die Frage, in welchen Settings, verstanden als situativer Rahmen des Methodeneinsatzes, Methoden, die im Geiste des klassischen analytisch-rationalen Planungsmodells entstanden, im Sinne des „Communicative Turn“ weiterentwickelt werden können, um ihre Potenziale zu erhalten, gleichzeitig aber ihre Defizite auszugleichen. Für die etablierte Methode der Nutzwertanalyse wurde in den Experimenten gezeigt, dass sie durchaus auch eine kommunikative Methode ist. Was ihren Einsatz in explizit kommunikativen Gruppenprozessen von Akteuren angeht, liegen jedoch in der Planungsforschung kaum Erkenntnisse vor. Die systematischere Einbeziehung kommunikativer und emotional-kreativer Aspekte in etablierte ,rationale ' Planungsmethoden sowie die Integration unterschiedlicher Methodentypen ist ein vielversprechendes zukünftiges Forschungsfeld. Für weitere Forschungen hat sich die Anwendung von Laborexperimenten, trotz deren dargestellter Defizite, grundsätzlich bewährt. In weiteren Untersuchungen werden nunmehr Planungsmethoden in kontrolliert unterschiedlichen kommunikativen Rahmen angewendet und der Einfluss dieser unterschiedlichen Settings auf die Effektivität der Methoden bewertet. Für die planungstheoretische Debatte ist die hohe Bedeutung von Kreativitätsmethoden im Planungsalltag ein Hinweis darauf, dass es lohnend ist, neben der rationalen und kommunikativen auch die kreativ-emotionale Dimension stärker einzubeziehen.

\section{Literatur}

Alexander, E. R. (1984): After Rationality, What? A Review of Responses to Paradigm Breakdown. In: Journal of the American Planning Association 50, 1, 62-69. doi: 10.1080/ 01944368408976582

Bäcker, A. (1996): Rationalität als Grundproblem der strategischen Unternehmensplanung. Ein Beitrag zur Erklärung und Überwindung der Rationalitätskrise in der Planungstheorie. Wiesbaden.
Baum, H. S. (1996): Why the Rational Paradigm Persists: Tales from the Field. In: Journal of Planning Education and Research 15, 2, 127-135. doi: 10.1177/0739456X9601500205

Bechmann, A. (1978): Nutzwertanalyse, Bewertungstheorie und Planung. Bern/Stuttgart. = Beiträge zur Wirtschaftspolitik 29.

Bechmann, A.; Kiemstedt, H. (1974): Die Landschaftsbewertung für das Sauerland als ein Beitrag zur Theoriediskussion in der Landschaftsplanung. In: Raumforschung und Raumordnung 32, 5, 190-202.

Beck, S. M. (2011): Partizipative Planungsinstrumente für eine nachhaltige und multifunktionale Waldbewirtschaftung. Vergleichende Anwendung und Nutzerevaluation des analytisch hierarchischen Prozesses und der Nutzwertanalyse. Freiburg. = Schriftenreihe Freiburger Forstliche Forschung 49.

Bischoff, A.; Selle, K.; Sinning, H. (2005): Informieren. Beteiligen. Kooperieren. Eine Übersicht zu Formen, Verfahren, Methoden und Techniken. Dortmund.

Bortz, J.; Döring, N. (2016): Forschungsmethoden und Evaluation in den Sozial- und Humanwissenschaften. Berlin/Heidelberg.

Bryson, J. M. (2003): Strategic Planning and Management. In: Peters, B. G.; Pierre, J. (Hrsg.): Handbook of Public Administration. London, 38-47. doi: 10.4135/9781848608214.n4

Dalton, L. C. (1986): Why the Rational Paradigm Persists - The Resistance of Professional Education and Practice to Alternative Forms of Planning. In: Journal of Planning Education and Research 5, 3, 147-153. doi: $10.1177 / 0739456$ X6600500302

Diller, C. (2010): Methoden in der Praxis der deutschen Raumplanung. Überlegungen zur Systematisierung und Ergebnisse einer bundesweiten Umfrage in Raumplanungsinstitutionen. In: disP The Planning Review 46, 182, 36-49.

Diller, C.; Karic, S.; Oberding, S. (2017): Planungsmodelle und Planungsmethoden: Anhaltspunkte zur Strukturierung und Gestaltung von Planungsprozessen. In: Standort. Zeitschrift für Angewandte Geographie 41, 2, 74-79.

Diller, C.; Oberding, S. (2017): Der „Strategic Choice Approach“: ein in Deutschland unterschätzter Methodenbaukasten für die Raumplanung. In: disP - The Planning Review 53, 2, 94-108. doi: $10.1080 / 02513625.2017 .1341200$

Elster, J. (1982): Rationality. In: Floistad, G. (Hrsg.): Philosophy of science. Den Haag, 111-131. = Contemporary philosophy. A new survey 2.

Friend, J.; Hickling, A. (2005): Planning under Pressure. The Strategic Choice Approach. Amsterdam.

Fuchs, O. (2001): Der Strategic Choice Approach - Entscheidungsorientierter Planungsansatz zur Lösung komplexer Planungsaufgaben. In: Fürst, D.; Scholles, F. (Hrsg.): Handbuch Theorien und Methoden der Raum- und Umweltplanung. Dortmund, 130-138.

Fürst, D.; Scholles, F. (Hrsg.) (2008a): Handbuch Theorien und Methoden der Raum- und Umweltplanung. Dortmund.

Fürst, D.; Scholles, F. (2008b): Die Nutzwertanalyse und ihre Weiterentwicklung. In: Fürst, D.; Scholles, F. (Hrsg.): Handbuch Theorien und Methoden der Raum- und Umweltplanung. Dortmund, 431-451.

Ganser, K.; Siebel, W.; Sieverts, T. (1993): Die Planungsstrategie der IBA Emscher Park. Eine Annäherung. In: RaumPlanung 61, 112118.

Gill, L.; Lange, E.; Morgan, E.; Romano, D. (2013): An Analysis of Usage of Different Types of Visualisation Media within a Collaborative Planning Workshop Environment. In: Environment and Planning B 40, 4, 742-754. doi: 10.1068/b38049

Goleman, D. (1996): Emotionale Intelligenz. München.

Healey, P. (1992): Planning through Debate: The Communicative Turn in Planning Theory. In: The Town Planning Review 63, 2, 143 162.

Hemberger, C. (2014): Erwerb kognitiver und methodischer Handlungskompetenzen zur Bearbeitung komplexer Planungsprobleme. Entwicklung und Evaluation eines transdisziplinären Trai- 
ningsprogramms am Beispiel raumbezogener Aufgaben. München.

Hoffmann, A.; Oberding, S. (2017): Zur Systematisierung von Planungsprozessen. In: disP - The Planning Review 53, 3, 58-70. doi: $10.1080 / 02513625.2017 .1380426$

Jetter, F.; Skrotzki, R. (2005): Soziale Kompetenz: Führungskräfte lernen Emotionale Intelligenz, Motivation, Coaching. Regensburg/ Berlin.

Julian, D. A.; Reischl, T. M.; Carrick, R. V.; Katrenich, C. (1997): Citizen Participation - Lessons from a Local United Way Planning Process. In: Journal of the American Planning Association 63, 3, 345-355. doi: 10.1080/01944369708975927

Kanitsar, G.; Kittel, B. (2015): Experimentelle Methoden. In: Wenzelburger, G.; Zohlnhöfer, R. (Hrsg.): Handbuch Policy-Forschung. Wiesbaden, 379-407. doi: 10.1007/978-3-658-01968-6

Kersting, M.; Werbeck, N. (2014): Angewandte Nutzwertanalyse in Planung und Verwaltung. Essen. $=$ Dynaklim Kompakt 16.

Kou, G.; Ergu, D.; Peng, Y.; Shi, Y. (2013): Data Processing for the AHP/ANP. Berlin/Heidelberg. doi: 10.1007/978-3-642-29213-2

Lenk, H.; Spinner, H. (1989): Rationalitätstypen, Rationalitätskonzepte und Rationalitätstheorien im Überblick. In: Stachowiak, H. (Hrsg.): Allgemeine philosophische Pragmatik. Hamburg, 1-31. $=$ Handbuch pragmatischen Denkens 3 .

Lenk, T.; Oelsner, A.; Reimann, S. B. (2010): Von der Nutzwertanalyse zum Kulturranking - Erfahrungen am Beispiel der Messestadt Leipzig. Leipzig. = Arbeitspapiere des Lehrstuhls Finanzwissenschaft der Universität Leipzig 42.

Meise, J.; Volwahsen, A. (1980): Stadt- und Regionalplanung. Ein Methodenhandbuch. Braunschweig.

Nollau, H.-G.; Gottfried, U. (2009): Entscheidungskompetenz durch Anwendung der Vektor-Nutzwertanalyse. Lohmar.

Pommer, A. (2007): Entscheidungsunterstuitzung in der Projektentwicklung. Weimar. $=$ Schriftenreihe Bau- und Immobilienmanagement 6.

Rittel, H. W. J. (1992): Planen, Entwerfen, Design. Ausgewählte Schriften zu Theorie und Methodik. Stuttgart/Berlin/Köln.

Rommelfanger, H. J.; Eickemeier, S. H. (2002): Entscheidungstheorie. Klassische Konzepte und Fuzzy-Erweiterungen. Heidelberg.

Rost, D. H. (2010): Soziale Intelligenz, Emotionale Intelligenz, Praktische Intelligenz: Alternativen zum IQ? In: Rost, D. H. (Hrsg.): Intelligenz, Hochbegabung, Vorschulerziehung, Bildungsbenachteiligung. Münster, 37-83.

Saaty, T.L. (1977): A scaling method for priorities in hierarchical structures. In: Journal of Mathematical Psychology 15, 3, 234-281. doi: 10.1016/0022-2496(77)90033-5

Schäfer, C.; Witte, F. (2014): Wirtschaftlichkeitsuntersuchungen in Kommunen. Erstellung, Prüfung, Interpretation. Berlin. = Kommunale Verwaltungssteuerung 9.

Schönwandt, W. (2002): Planung in der Krise? Theoretische Orientierungen für Architektur, Stadt- und Raumplanung. Stuttgart.

Schubert, A. (2014): Emotionale Rationalität und Planung: Planungsansätze einer ,3. Generation`. Oder: Zum depolitisierenden Po- tenzial von Vertrauensbildung und Selbstversicherung. In: Sub \ urban: Zeitschrift für Kritische Stadtforschung 2, 1, 71-94.

Shipley, R.; Michela, J. L. (2006): Can vision motivate planning action? In: Planning Practice and Research 21, 2, 223-244. doi: 10.1080/02697450600944715

Siebel, W. (2006): Wandel, Rationalität und Dilemmata der Planung. In: Selle, K. (Hrsg.): Zur räumlichen Entwicklung beitragen. Konzepte. Theorien. Impulse. Dortmund, 195-209. = Planung neu denken 1 .

Siebert, J. (2009): Modellierung komplementärer Interaktionen. Konzeption eines innovativen Ansatzes in der multiattributiven Nutzentheorie. Siegburg.

Simon, H. A. (1991): Bounded Rationality and Organizational Learning. In: Organization Science 2, 1, 125-134. doi:10.1287/orsc.2.1. 125

Stamps, A.; Nasar, J. L.; Hanyu, K. (2005): Using Pre-construction Validation to Regulate Urban Skylines. In: Journal of the American Planning Association 71, 1, 73-91. doi: 10.1080/0194436050897 6406

Sternberg, R. J. (2002): Countdown zum Erfolg. Was man braucht, um seine Ziele wirklich zu erreichen. München.

Süß, H.-M.; Beauducel, A. (2011): Intelligenztests und ihre Bezüge zu Intelligenztheorien. In: Hornke, L. F.; Amelang, M.; Kersting, M. (Hrsg.): Leistungs-, Intelligenz- und Verhaltensdiagnostik. Göttingen, 97-234. = Enzyklopädie der Psychologie, Serie 2: Psychologische Diagnostik 3.

Te Brömmelstroet, M. (2015): A Critical Reflection on the Experimental Method for Planning Research. Testing the added value of PSS in a controlled environment. In: Planning Practice and Research 30, 2, 179-201. doi: 10.1080/02697459.2015.1023077

Vohl, H.-J. (2004): Balanced Scorecard im Mittelstand. Hamburg.

von Kanitz, A. (2015): Emotionale Intelligenz. Freiburg.

Weber, J. (2004): Das Scoring-Modell. Norderstedt.

Wiechmann, T. (2008): Planung und Adaption. Strategieentwicklung in Regionen, Organisationen und Netzwerken, Dortmund.

Wiechmann, T.; Hutter, G. (2010): Strategische Planung - Zum Stand der Diskussion. In: Hutter, G.; Wiechmann, T. (Hrsg.): Strategische Planung. Zur Rolle der Planung in der Strategieentwicklung für Städte und Regionen. Berlin, 7-16. = Planungsrundschau 18.

Winkler, S.; König, C. J.; Kleinmann, M. (2010): Single-Attribute Utility Analysis may be futile, but this can't be the end of the Story: Causal Chain Analysis as an Alternative. In: Personnel Psychology 63, 4, 1041-1065. doi: 10.1111/j.1744-6570.2010.01197.x

Zampich, T. (2002): Bewertung von Eignungsflächen für Windkraftanlagen. Dargestellt am Beispiel von ausgewählten Gemeinden des Regierungsbezirks Münster. Hamburg.

Zangemeister, C. (1970): Nutzwertanalyse in der Systemtechnik. Eine Methodik zur multidimensionalen Bewertung und Auswahl von Projektalternativen. Berlin. 